\title{
Memantine for the treatment of frontotemporal dementia: a meta-analysis
}

\author{
This article was published in the following Dove Press journal: \\ Neuropsychiatric Disease and Treatment \\ 12 November 2015 \\ Number of times this article has been viewed
}

Taro Kishi

Shinji Matsunaga

Nakao Iwata

Department of Psychiatry, Fujita Health University School of Medicine, Toyoake, Japan
Correspondence: Taro Kishi

Department of Psychiatry, Fujita Health

University School of Medicine, I-98

Dengakugakubo, Kutsukakecho Toyoake,

Aichi 470-I 192, Japan

$\mathrm{Tel}+8 \mid 562939250$

Fax +8| $56293|83|$

Email tarok@fujita-hu.ac.jp
Background: There is no conclusive evidence supporting the efficacy of memantine in frontotemporal dementia (FTD). We conducted a comprehensive meta-analysis of memantine concerning the efficacy and tolerability of memantine in FTD.

Methods: Studies were identified through searches of PubMed, databases of the Cochrane Library, and PsycINFO citations up to April 10, 2015. Outcomes were Clinical Global Impression (primary), Mini-Mental State Examination, Neuropsychiatric Inventory, and Zarit Burden Interview scores as well as all-cause discontinuation. Standardized mean difference and risk ratio with $95 \%$ confidence interval were calculated.

Results: Two randomized controlled trials (RCTs) (total $n=130$ ) met the inclusion criteria. Memantine was marginally superior to placebo as assessed by the Clinical Global Impression scores ( standardized mean difference $=-0.34,95 \%$ confidence interval $=-0.68-0.01, P=0.06$ ). However, there were no significant differences in Mini-Mental State Examination, Neuropsychiatric Inventory, and Zarit Burden Interview scores as well as all-cause discontinuation between memantine and placebo.

Conclusion: Our results suggest that memantine may benefit FTD patients. However, because only two randomized controlled trials have addressed this issue, further studies using larger samples are needed.

Keywords: frontotemporal dementia, memantine, meta-analysis, Clinical Global Impression

\section{Introduction}

Memantine is considered to act as a noncompetitive inhibitor of $N$-methyl D-aspartate receptors that may be overactivated in a variety of neurodegenerative diseases, including frontotemporal dementia (FTD). ${ }^{1}$ A recent review ${ }^{2}$ of two double-blind, randomized controlled trials (RCTs) ${ }^{3,4}$ concluded that memantine does not benefit patients with FTD. However, this was a systematic review rather than a meta-analysis of pooled patient groups. Therefore, negative results in these separate RCTs may be due to insufficient sample sizes for detecting small differences in outcome between memantine and placebo. A meta-analysis can increase the statistical power for group comparisons and overcome the limitation of sample size in underpowered studies. ${ }^{3-5}$ Moreover, using standardized mean difference (SMD) analyses, outcomes with different metrics can be combined. ${ }^{5}$ We conducted a meta-analysis of these two double-blind RCTs $\mathrm{s}^{3,4}$ to examine whether memantine is beneficial for the treatment of FTD.

\section{Methods}

This meta-analysis was performed according to the Preferred Reporting Items for Systematic Reviews and Meta-Analyses guidelines. ${ }^{6}$ 


\section{Inclusion criteria, search strategy, data extraction, and outcomes}

Inclusion criteria were double-blind RCTs of memantine for patients with FTD. Studies were identified through searches of PubMed, databases of the Cochrane Library, and PsycINFO citations up to April 10, 2015. The following English keywords were used without language restriction: "memantine" AND "randomized" OR "random" OR "randomly" AND "frontotemporal dementia". Two authors (TK and SM) independently extracted, checked, and entered the data into Review Manager (Version 5.3 for Windows, Cochrane Collaboration, London, UK, http://tech.cochrane.org/Revman).

\section{Data synthesis and statistical analysis}

The primary outcome was Clinical Global Impression score. We combined data from Clinician's Interview-Based Impression of Change Plus Caregiver Input ${ }^{4}$ with that from Clinical Global Impression of Change. ${ }^{3}$ Secondary outcomes were total scores on the Mini-Mental State Examination (MMSE), Neuropsychiatric Inventory (NPI), and Zarit Burden Interview (ZBI) as well as all-cause discontinuation. We allowed only intention-to-treat (ITT) or modified ITT data (ie, at least one dose or at least one follow-up assessment). For continuous data, SMD was used, combining the effect-size (Hedges'g) data. For dichotomous data, the relative risk (RR) was estimated along with associated 95\% confidence interval $(95 \% \mathrm{CI})$. Overall, SMD and RR with 95\% CI were estimated with Mantel-Haenszel fixedeffects $^{7}$ or DerSimonian-Laird random-effects models. ${ }^{8}$ The random-effects model is more conservative than the fixed-effects model and produces a wider CI. When there is no evidence of heterogeneity between studies, the randomeffects model will produce similar results to the fixed-effects model. Therefore, when it was confirmed that there was no heterogeneity, we calculated pooled SMD and RR according to the Mantel-Haenszel fixed-effects model. If there was evidence of heterogeneity, we calculated pooled SMD and RR according to the DerSimonian-Laird random-effects model. The Cochrane risk of bias criteria (Cochrane Collaboration, http://www.cochrane.org/) was used to perform methodological quality control. As only two studies were included, we did not confirm the possibility of publication bias by Funnel plot.

\section{Results \\ Study characteristics}

The search using the chosen keywords yielded eight references. We excluded five references, including one duplicate reference, based on title and abstract review (one reference is a single-arm study and other three references are not clinical trials). One reference was excluded based on full-text evaluation because it was a systematic review article. ${ }^{2}$ Two RCTs $(n=130)$ were included in our meta-analysis. ${ }^{3,4}$ No additional articles were identified by manually searching all article references. Both RCTs were double-blind, mentioned the required study design details, and used ITT or modified ITT data. The study duration was 26 weeks in one ${ }^{3}$ and 52 weeks in the other. ${ }^{4}$ Vercelletto et $\mathrm{al}^{4}$ reported that all patients were Caucasian, the mean MMSE baseline scores for all patients was 24.8, and the mean NPI baseline scores for all patients was 29.5. Boxer et $\mathrm{al}^{3}$ reported that the mean MMSE baseline scores in memantine and placebo groups were 24.3 and 25.1, respectively, and the mean NPI baseline scores in memantine and placebo groups were 20.6 and 21.5 , respectively.

\section{The results of meta-analysis}

Memantine was marginally superior to placebo as indicated by the Clinical Global Impression scores (SMD $=-0.34$, $95 \% \mathrm{CI}=-0.68-0.01, P=0.06, \mathrm{I}^{2}=0 \%$ ) (Table 1). However, there were no significant differences in MMSE, NPI, and ZBI total scores. There was no difference in all-cause discontinuation between memantine and placebo treatment groups (Table 1).

\section{Discussion}

To the best of our knowledge, this is the first comprehensive meta-analysis of memantine for the treatment of FTD. Antidepressants such as paroxetine and trazodone as well as methylphenidate, dextroamphetamine, and oxytocin were reported to improve behavioral symptoms in FTD patients. ${ }^{2}$ However,

Table I The results of meta-analysis

\begin{tabular}{llllll}
\hline Outcomes & Number of patients & $\mathbf{I}^{2}$ & Standardized mean difference & 95\% confidence interval & $P$-value \\
\hline Clinical Global Impression & 129 & 0 & -0.33 (fixed model) & -0.68 to 0.01 & 0.06 \\
Mini-Mental State Examination & 122 & 14 & -0.06 (fixed model) & $-0.4 I$ to 0.30 & 0.76 \\
Neuropsychiatric Inventory & 120 & 0 & -0.23 (fixed model) & -0.59 to 0.13 & 0.22 \\
Zarit Burden Interview & 120 & 0 & -0.25 (fixed model) & $-0.6 I$ to $0.1 \mathrm{I}$ & 0.18 \\
& & & Risk ratio & & \\
All-cause discontinuation & 130 & 0 & I.II (fixed model) & $0.38-3.22$ & 0.85 \\
\hline
\end{tabular}


none of the RCTs showed an improvement in cognitive function with memantine. Our meta-analysis showed that memantine was marginally superior to placebo as assessed by the Clinical Global Impression scores (SMD $=-0.34,95 \%$ $\mathrm{CI}=-0.68$ to $\left.0.01, P=0.06, \mathrm{I}^{2}=0 \%\right)$. Although memantine was not statistically superior to placebo as assessed by MMSE, NPI, and ZBI total scores, memantine did show favorable results according to NPI and ZBI total scores compared with placebo. These lack of associations may depend on the small sample size, which limited the power of the study (two RCTs $[n=130])$. Therefore, this result suggests that memantine may be beneficial for the treatment of FTD. Moreover, there was no significant difference in all-cause discontinuation in either study, suggesting that memantine was well tolerated. This small meta-analysis suggests possible modest benefits of memantine for general dysfunction in FTD without severe side effects. Several limitations to the present analysis have to be noted. First of all, the number of studies included in this meta-analysis was small (two RCTs $[n=130]$ ). Therefore, we failed to take into account the confounding variables (eg, funding, trial design, and ethical issue) and did not try to eliminate any other factors. FTD is also considered to be a genetically and pathologically heterogeneous disorder. ${ }^{9}$ Large-scale studies are warranted to identify patient subgroups showing the most robust response to memantine.

\section{Conclusion}

There were no significant differences in the Clinical Global Impression, MMSE, NPI, and ZBI total scores between memantine and placebo treatment groups. However, the result of Clinical Global Impression scores was marginally significant (SMD $=-0.34, P=0.06)$. However, because only two RCTs have addressed this issue, the lack of associations might depend on the small sample size, which limited the power of the study. Therefore, further studies using larger samples are needed.

\section{Author contributions}

All authors had full access to all study data and are responsible for the integrity of the data and the accuracy of any data analysis. All authors drafted the final manuscript. All authors contributed toward data analysis, drafting and revising the paper and agree to be accountable for all aspects of the work.

\section{Disclosure}

Dr Kishi has received speaker's honoraria from Abbott, Astellas, Daiichi-Sankyo, Dainippon Sumitomo, Eisai, Eli Lilly, GlaxoSmithKline, Yoshitomi, Otsuka, Meiji, Shionogi, Janssen, Novartis, Tanabe-Mitsubishi, and Pfizer. Dr Matsunaga has received speaker's honoraria from Eisai, Janssen, Novartis, Daiichi-Sankyo, Ono, Eli Lilly, Takeda, and Otsuka. Dr Iwata has received speaker's honoraria from Astellas, Dainippon Sumitomo, Eli Lilly, GlaxoSmithKline, Janssen, Yoshitomi, Otsuka, Meiji, Shionogi, Novartis, and Pfizer and has received research grant from GlaxoSmithKline and Otsuka. The authors report no other conflicts of interest in this work.

\section{References}

1. Kalia LV, Kalia SK, Salter MW. NMDA receptors in clinical neurology: excitatory times ahead. Lancet Neurol. 2008;7(8):742-755.

2. Nardell M, Tampi RR. Pharmacological treatments for frontotemporal dementias: a systematic review of randomized controlled trials. Am J Alzheimers Dis Other Demen. 2014;29(2):123-132.

3. Boxer AL, Knopman DS, Kaufer DI, et al. Memantine in patients with frontotemporal lobar degeneration: a multicentre, randomised, doubleblind, placebo-controlled trial. Lancet Neurol. 2013;12(2):149-156.

4. Vercelletto M, Boutoleau-Bretonniere C, Volteau C, et al. Memantine in behavioral variant frontotemporal dementia: negative results. J Alzheimers Dis. 2011;23(4):749-759.

5. Higgins J, Green S. Cochrane Handbook for Systematic Reviews of Interventions Version 5.1.0. The Cochrane Collaboration. 2011. Available from: www.cochrane-handbook.org. Accessed November 6, 2015.

6. Moher D, Liberati A, Tetzlaff J, Altman DG; PRISMA Group. Preferred reporting items for systematic reviews and meta-analyses: the PRISMA statement. BMJ. 2009;339:b2535

7. Mantel N, Haenszel W. Statistical aspects of the analysis of data from retrospective studies of disease. J Natl Cancer Inst. 1959;22(4):719-748.

8. Higgins JP, Thompson SG, Deeks JJ, Altman DG. Measuring inconsistency in meta-analyses. BMJ. 2003;327(7414):557-560.

9. Benussi A, Padovani A, Borroni B. Phenotypic heterogeneity of monogenic frontotemporal dementia. Front Aging Neurosci. 2015;7:171.
Neuropsychiatric Disease and Treatment

\section{Publish your work in this journal}

Neuropsychiatric Disease and Treatment is an international, peerreviewed journal of clinical therapeutics and pharmacology focusing on concise rapid reporting of clinical or pre-clinical studies on a range of neuropsychiatric and neurological disorders. This journal is indexed on PubMed Central, the 'PsycINFO' database and CAS,

\section{Dovepress}

and is the official journal of The International Neuropsychiatric Association (INA). The manuscript management system is completely online and includes a very quick and fair peer-review system, which is all easy to use. Visit http://www.dovepress.com/testimonials.php to read real quotes from published authors. 